\title{
BMJ Open Study protocol and implementation details for a pragmatic, stepped-wedge cluster randomised trial of a digital adherence technology to facilitate tuberculosis treatment completion
}

To cite: Crowder R, Kityamuwesi A, Kiwanuka N, et al. Study protocol and implementation details for a pragmatic, stepped-wedge cluster randomised trial of a digital adherence technology to facilitate tuberculosis treatment completion. BMJ Open 2020;10:e039895. doi:10.1136/ bmjopen-2020-039895

- Prepublication history for this paper is available online. To view these files, please visit the journal online (http://dx.doi. org/10.1136/bmjopen-2020039895).

$\mathrm{RC}$ and $\mathrm{AK}$ contributed equally.

Received 29 April 2020

Revised 09 November 2020

Accepted 11 November 2020

Check for updates

(c) Author(s) (or their employer(s)) 2020. Re-use permitted under CC BY-NC. No commercial re-use. See rights and permissions. Published by BMJ.

For numbered affiliations see end of article.

Correspondence to Dr Achilles Katamba axk95@case.edu

\section{ABSTRACT}

Introduction Low-cost digital adherence technologies (DATs) such as 99D0TS have emerged as an alternative to directly observed therapy (DOT), the current standard for tuberculosis (TB) treatment supervision. However, there are limited data to support DAT scale-up. The 'DOT to DAT' trial aims to evaluate the effectiveness and implementation of a 99D0TS-based TB treatment supervision strategy.

Methods and analysis This is a pragmatic, steppedwedge cluster randomised trial, with hybrid type 2 effectiveness-implementation design. The trial will include all adults (estimated $\mathrm{N}=1890$ ) treated for drug-susceptible pulmonary TB over an 8-month period at 18 TB treatment units in Uganda. Three sites per month will switch from routine care (D0T) to the intervention (99D0TS-based treatment supervision) beginning in month 2 , with the order determined randomly. 99D0TS enables patients to be monitored while self-administering TB medicines. Patients receive daily automated short message service (SMS) dosing reminders and confirm dosing by calling toll-free numbers. The primary effectiveness outcome is the proportion of patients completing TB treatment. With 18 clusters randomised into six steps and an average cluster size of 15 patients per month, the study will have $89 \%$ power to detect a $10 \%$ or greater increase in treatment completion between the routine care and intervention periods. Secondary outcomes include more proximal effectiveness measures as well as quantitative and qualitative assessments of the reach, adoption and implementation of the intervention.

Ethics and dissemination Ethics approval was granted by institutional review boards at Makerere University School of Public Health and the University of California San Francisco. Findings will be disseminated through peer-reviewed publications, presentations at scientific conferences and presentations to key stakeholders. Trial registration number PACTR201808609844917.
Strengths and limitations of this study

- This is the first randomised trial to evaluate a 99D0TS-based strategy for tuberculosis treatment supervision.

- The intervention was designed using the Predisposing, Reinforcing, and Enabling Constructs in Educational Diagnosis and Evaluation (PRECEDE) framework and adapted for local context in Uganda using human-centred design.

- Trial outcomes were selected using the Reach, Effectiveness, Adoption, Implementation and Maintenance (RE-AIM) framework.

- The intervention was implemented by health facility staff and will be assessed using routinely collected data to approximate the real-world impact of this intervention.

Adherence is not a primary outcome of this trial.

\section{INTRODUCTION}

Tuberculosis (TB) is the leading infectious cause of death worldwide despite being a preventable and curable disease. ${ }^{1}$ Poor adherence to medication continues to be a major obstacle to TB elimination, resulting in prolonged infectiousness, emergence of multidrug resistance and increased risk of poor treatment outcomes. The WHOrecommended strategy, directly observed therapy short-course (DOTS), has been the standard of care for TB treatment supervision since the 1990s. As part of this strategy, a health worker is expected to observe patients swallow each dose of anti-TB medication. However, directly observed therapy (DOT) is time-consuming, costly and inconvenient for 
both patients and providers, while its implementation is also difficult to monitor. ${ }^{23}$ Not surprisingly, TB treatment success rates remain below the $90 \%$ target in most highburden countries, despite reported DOT coverage over $90 \% .^{1}$

With the increase in mobile phone penetration among patients and healthcare workers in high TB burden countries, ${ }^{4}$ digital adherence technologies (DATs) could address some of the challenges associated with implementation of the DOTS strategy in a patient-centred manner. To date, such technologies have received only a conditional recommendation by the WHO due to very low quality of evidence. ${ }^{5}$ The first randomised trial of a DAT found that short message service (SMS) reminders alone did not improve medication adherence, but a medication event reminder monitor did. ${ }^{6}$ However, treatment outcomes did not improve, and both trials and programmatic research have shown variable uptake of DAT. ${ }^{7-10}$

The 'DOT to DAT' trial aims to evaluate the effectiveness, implementation and cost-effectiveness of a culturally and contextually adapted version of 99DOTS in Uganda. 99DOTS is a low-cost DAT wherein patients self-report medication adherence by calling toll-free phone numbers hidden underneath pills in blister packs. ${ }^{11}$ We present the research methods used for the first randomised trial to evaluate the effectiveness and implementation of a 99DOTS-based strategy for TB treatment supervision in routine care settings.

\section{Conceptual basis for trial design}

Numerous conceptual frameworks have emerged to improve the implementation and success of health interventions through theory-based design and evaluation. The Predisposing, Reinforcing, and Enabling Constructs in Educational Diagnosis and Evaluation (PRECEDE) framework $^{12}$ guided the development of the 99DOTSbased intervention strategy, while the Reach, Effectiveness, Adoption, Implementation, and Maintenance (RE-AIM) framework guided its comprehensive evaluation. Behaviour change models including the Theoretical Domains Framework ${ }^{13} 14$ and the Unified Theory of Acceptance and Use of Technology ${ }^{15} 16$ guided the assessment and analysis of patient-level and provider-level barriers to adoption and implementation of 99DOTS.

The PRECEDE framework emphasises the need for multifaceted health promotion interventions to address predisposing, enabling and reinforcing factors in order to achieve behaviour change. ${ }^{12}$ The 99DOTS-based intervention was designed to address key predisposing (social isolation and the high direct and indirect cost of clinic visits), enabling (inability of health facility staff to focus limited time and resources on non-adherent patients) and reinforcing (a lack of real-time information on patient adherence to medications) factors related to TB treatment adherence identified through the literature and formative human factors research that preceded the trial. $^{11}$
The RE-AIM framework was designed to ensure that trials assess not only the effect of interventions but also their translatability and public health impact. ${ }^{17}{ }^{18}$ This hybrid type 2 trial therefore focuses on the effectiveness of the 99DOTS-based intervention in improving TB treatment outcomes, but concurrently examines its reach into the target population, adoption by target settings and staff, and implementation fidelity and costs. ${ }^{19}$ The goal of assessing translatability and public health impact is further enhanced through pragmatic design choices for each element of the trial in order to maximise applicability in real-world settings. ${ }^{20}$

\section{METHODS AND ANALYSIS}

\section{Study aims}

The primary aim of the trial is to determine whether a 99DOTS-based strategy increases the proportion of patients who complete TB treatment in comparison to routine care. Secondary aims include (1) comparing short-term treatment outcomes and loss to follow-up between the intervention and routine care arms; (2) assessing the reach, adoption and implementation of the 99DOTS-based strategy; and (3) evaluating the incremental costs and cost-effectiveness of the 99DOTSbased strategy as compared with routine care from the health system perspective. Our primary hypothesis is that 99DOTS-based TB treatment supervision will improve TB treatment outcomes. Our secondary hypotheses are that the 99DOTS-based strategy will have high uptake among patients and providers, and be cost-effective.

\section{Study design}

This is a pragmatic, stepped-wedge randomised trial, with a hybrid type 2 implementation-effectiveness design. ${ }^{19}$ We included repeated cross-sectional samples of eligible individuals initiating TB treatment at participating health facilities at eight time points (months). Patient outcomes were assigned to the health facility and month in which they initiated treatment. As depicted in figure 1, the intervention strategy was sequentially introduced at three sites each in months $2-7$, with all sites under routine care in month 1 and all sites using the intervention strategy in month 8. Following implementation of the intervention at each site, health facility staff were instructed to offer 99DOTS to all new eligible patients initiating treatment and to continue supervising patients already on treatment using routine care. The month of switch from control to intervention (buffer period) will be excluded from analysis. We chose the stepped-wedge trial design to maximise equity and acceptability (all sites will receive the intervention), minimise logistical constraints associated with introducing the intervention simultaneously at a large number of sites, account for secular outcome trends and allow for iterative learning and changes in intervention roll-out as would occur during eventual scale-up. We chose a highly pragmatic approach to trial implementation, as described 


\begin{tabular}{|l|l|l|l|l|l|l|l|l|}
\multicolumn{1}{|c|}{ Month } \\
\hline Block 1 & 1 & 2 & 3 & 4 & 5 & 6 & 7 & 8 \\
\hline Block 2 & & & & & & & & \\
\hline Block 3 & & & & & & & & \\
\hline Block 4 & & & & & & & & \\
\hline Block 5 & & & & & & & & \\
\hline Block 6 & & & & & & & & \\
\hline
\end{tabular}

\begin{tabular}{|l|l|}
\hline & Routine care (control period) \\
\hline & Switch to 99DOTS (buffer period) \\
\hline & 99DOTS implementation (intervention period) \\
\hline
\end{tabular}

Figure 1 99DOTS randomisation and enrolment schedule. The trial includes 18 health facilities divided into six equal size blocks (three health facilities per block). The health facilities all continue routine tuberculosis (TB) treatment supervision in month 1, begin switching to the 99DOTS-based intervention strategy in month 2 (one block per month in a random order), and all use the intervention strategy in month 8 .

in sections below, to understand the real-world impact of the 99DOTS-based intervention strategy.

\section{Study setting}

The trial is being conducted at $18 \mathrm{~TB}$ treatment units within hospitals and health centres across 15 districts of Uganda. The TB treatment units are affiliated with the Uganda National Tuberculosis and Leprosy Programme (NTLP) and provide TB treatment free of charge using a mix of facility-based and community-based DOTS.
Uganda was chosen as the trial setting due to its high TB burden (200 cases/100000 in 2018) and low treatment success rate (72\% in 2017). ${ }^{1}$ Previous studies have found $69 \%-75 \%$ of TB patients in Uganda have access to a phone. ${ }^{21} 22$

Study sites were selected after reviewing 2016 TB treatment outcomes and 2017 TB case finding data reported to the NTLP from all 1514 registered TB treatment units. We included treatment units that (1) diagnosed $>10$ patients with pulmonary TB/month in 2017, (2) were not located within Kampala District (many active ongoing projects in this district), (3) were located within $225 \mathrm{~km}$ of Kampala City (for feasibility) and (4) had a pulmonary TB treatment success rate in $2016<80 \%$ (to be able to show an impact).

Of the 1514 treatment units registered with the Uganda NTLP, 23 treatment units met our inclusion criteria (figure 2$)$. The majority $(\mathrm{n}=1435)$ diagnosed fewer than 10 patients with TB/month in 2017, 17 are located within Kampala district, 31 are not within $225 \mathrm{~km}$ of Kampala district and 8 had a treatment success rate $>80 \%$ in 2016. Of the 23 remaining treatment units, we selected 18 located in 15 districts (10 in Central, 7 in Eastern and 1 in Western Uganda) in consultation with the NTLP. Project staff visited the Chief Administrative Officers, District Health Officers and Facility Directors for the 18 sites to discuss the study. All signed a memorandum of agreement for their site to participate in the study.
1514 TB treatment units assessed for eligibility (treatment units registered with NTLP)

18 TB treatment units randomised
1496 TB treatment units excluded:

- Treatment unit that treats fewer than 10 TB patients/month $(n=1435)$

- Treatment unit with Kampala District $(n=17)$

- Treatment unit not within $225 \mathrm{~km}$ of Kampala $(\mathrm{n}=31)$

- Treatment unit with treatment success rate $>80 \%(\mathrm{n}=8)$

- Treatment unit not selected in consultation with NTLP $(\mathrm{n}=5)$

Not in target population:

- $<18$ years old

- Extrapulmonary TB

- Drug-resistant TB
Target population: Adult patients started on drugsusceptible pulmonary TB treatment during the period 1 December 2018 - 31 July 2019
Study population: Adult patients started on drugsusceptible pulmonary TB treatment during the period 1 December 2018 - 31 July 2019, who did not transfer to another health facility during treatment (estimated $\mathrm{N}=1890$ )
Patients excluded if:

- Transferred out to another health facility during treatment

Figure 2 Flow diagram of tuberculosis (TB) treatment units and patients included in the directly observed therapy (DOT) to digital adherence technologies (DAT) trial. Eighteen TB treatment units were randomised, and all eligible patients initiating TB treatment at these facilities during the study period will be analysed. NTLP, National Tuberculosis and Leprosy Programme. 


\section{Study participants}

All adult patients treated for drug-susceptible pulmonary TB at participating treatment units during the study period will be eligible for inclusion. Children, patients treated for drug-resistant or extrapulmonary TB and patients transferred to another facility to complete treatment will be excluded. To be enrolled on 99DOTS, a patient must also have access to a phone. Surveys to assess 99DOTS implementation will be conducted in a random subset of eligible patients and all health workers involved in TB treatment supervision at each treatment unit. Timeand-motion studies to assess costs will also be conducted among health workers involved in TB treatment supervision at each treatment unit.

\section{Routine care and intervention strategies \\ Routine care}

The conventional approach to TB treatment supervision in Uganda is a mix of community-based and facilitybased DOT, as per NTLP guidelines. At the time of treatment initiation, treatment unit staff record patient demographic and clinical details in the NTLP register and are supposed to provide TB-focused counselling. Most patients with $\mathrm{TB}$ are treated using communitybased DOT, wherein they name a treatment supporter, a family member or non-family community member, and are provided with a 2-week supply of medicines in the first 2 months (intensive phase) and a 1-month supply of medicines in the next 4 months (continuation phase) of TB treatment. Patients take their medicines from home (with or without observation by a treatment supporter) and return to the health facility for medication refills. At each refill visit, health facility staff are supposed to assess adherence via patient self-report and provide additional counselling. Health facility staff are also supposed to call or visit patients who do not return for refills, but patient follow-up is limited across health facilities.

\section{Intervention}

The 99DOTS-based intervention strategy is comprised of four main components that address distinct barriers to successful TB treatment (table 1):

1. Daily automated SMS dosing reminders.

2. Daily dosing confirmation using toll-free phone calls.

3. Weekly automated interactive voice response (IVR) check-in phone calls.

4. Differential management protocol based on dosing history and response to IVR check-in.

Formative research using human-centred design was used to adapt the generic 99DOTS product to the local context, including changes to the envelope design and addition of a rotating series of educational/motivational audio messages heard when patients call in to report dosing (details of the formative research will be reported in a separate publication) (figure 3 ).

TB treatment unit staff will be requested to offer 99DOTS-based treatment supervision to all eligible patients initiating TB treatment, and to register patients

\begin{tabular}{|c|c|c|}
\hline \multicolumn{2}{|c|}{ Component } & \multirow{2}{*}{$\begin{array}{l}\text { Barrier addressed } \\
\text { Addresses high cost of clinic } \\
\text { visits for patients and assists with } \\
\text { memory and planning processes } \\
\text { known to be importance to } \\
\text { adherence. }\end{array}$} \\
\hline 1 & $\begin{array}{l}\text { Daily dosing } \\
\text { reminders via } \\
\text { automated } \\
\text { SMS }\end{array}$ & \\
\hline 2 & $\begin{array}{l}\text { Daily dosing } \\
\text { confirmation } \\
\text { via toll-free } \\
\text { phone calls }\end{array}$ & $\begin{array}{l}\text { Addresses high cost of clinic } \\
\text { visits for patients, lack of real-time } \\
\text { information for providers on patient } \\
\text { adherence to medications. }\end{array}$ \\
\hline 3 & $\begin{array}{l}\text { Weekly } \\
\text { check-in via } \\
\text { interactive } \\
\text { voice } \\
\text { response } \\
\text { phone calls }\end{array}$ & $\begin{array}{l}\text { Addresses lack of social support } \\
\text { and feeling of isolation during TB } \\
\text { treatment; shown to be effective } \\
\text { in other contexts at increasing } \\
\text { connection with community health } \\
\text { workers and reducing social } \\
\text { isolation. }\end{array}$ \\
\hline 4 & $\begin{array}{l}\text { Differential } \\
\text { management } \\
\text { protocol }\end{array}$ & $\begin{array}{l}\text { Addresses limited time and } \\
\text { resources among TB treatment unit } \\
\text { staff and the need to focus on non- } \\
\text { adherent patients. }\end{array}$ \\
\hline
\end{tabular}

TB, tuberculosis.

who accept 99DOTS-based treatment supervision on the 99DOTS platform via a mobile app. Given the pragmatic nature of the trial, the decision to offer and accept 99DOTS-based treatment supervision will be made by treatment unit staff and patients, respectively. Once registered, treatment unit staff will instruct patients on how to use the 99DOTS pill pack and make a call to the 99DOTS system using the toll-free number revealed when pills are pushed out of the medication blister pack (figure 3). Staff will also help patients personalise their 99DOTS pill pack by choosing a decorative cover, educational or motivational sticker for the inside flap, writing in their health worker's contact information and selecting a time of day to take their medication (figure 3).

When patients call to confirm dosing, they hear a recorded educational or motivational message about continuing and completing TB treatment. Each dose confirmed via phone call is logged by 99DOTS and reflected on the 99DOTS smartphone app dashboard, viewable by treatment unit staff. During routine drug refill visits (same schedule as described for routine care period), health facility staff are asked to review adherence data in the 99DOTS app before providing counselling. At the conclusion of the intensive phase of treatment, patients with adherence $>90 \%$ are offered the option of returning back to the health facility after 2 months instead of the standard 1 month.

Health facilities using 99DOTS to manage TB patient treatment will be provided one smartphone per facility, minimal funds to cover cell phone data and an average of 300000 USh (approximately US\$82) per month to facilitate patient follow-up. Treatment unit staff will not be 


\section{Original 99DOTS Envelope}

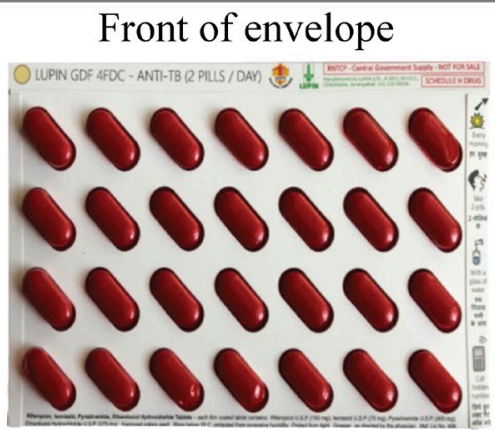

Back of envelope

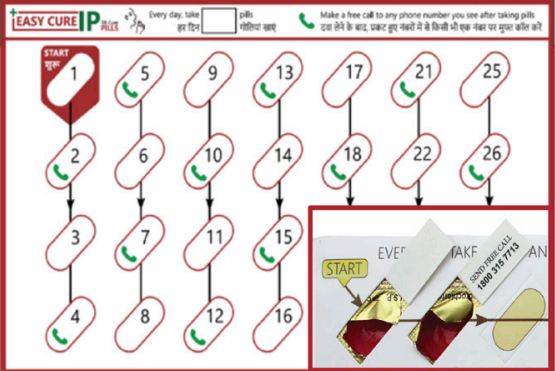

Adapted 99DOTS envelope
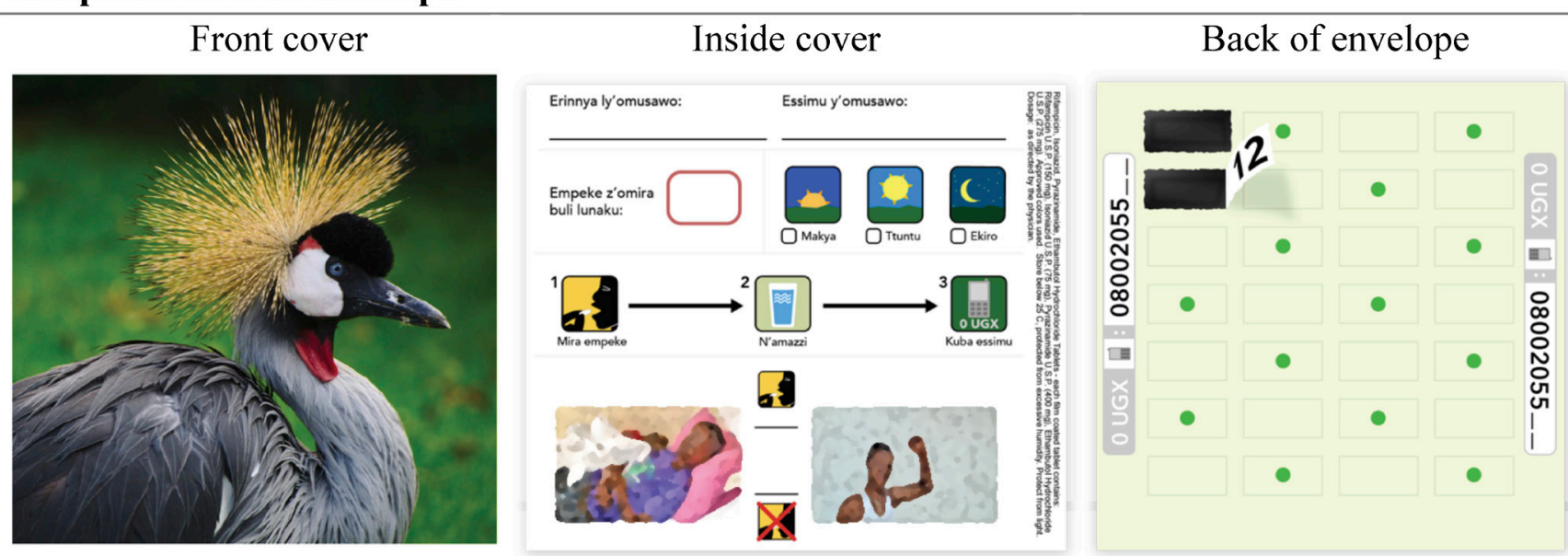

Figure 3 Original and adapted versions of the 99DOTS envelope. The original 99DOTS envelope was two-sided (top left and right). The original envelope was adapted using human-centred design to add a decorative front cover to hide pills (and thereby reduce potential stigma; bottom left); include space for writing in the health worker's name and phone number, simplified pictorial instructions for taking pills, and motivational imagery on the inside cover (bottom middle); and provide simplified guide to the order in which to take pills on the back cover (bottom right). In addition, the audio tone heard when patients make daily phone calls to report medication dosing was replaced with a rotating series of educational or motivational messages recorded by local health workers.

provided any extra compensation to reflect future conditions under eventual scale-up.

\section{Implementation considerations}

99DOTS training will occur at each health facility during the month in which it is scheduled to switch to the intervention. Project staff and the District TB Officer will conduct a 2-3 day training jointly at each health facility using standardised training materials. Treatment unit staff will be trained on how to register patients on the 99DOTS platform via a smartphone app, counsel patients regarding use of 99DOTS, use the 99DOTS application to review dosing history and conduct differential management based on dosing history and response to weekly check-ins. Mentored patient enrolment on 99DOTS will be conducted as part of the training.

During the trial, treatment unit staff will make all decisions regarding management of patients with TB in both the routine care and intervention periods. Research staff will not be onsite after training with the exception of 2-3 day quarterly site visits to resolve data cleaning queries and implement surveys and health economic substudies. Thus, the implementation is highly pragmatic and meant to approximate how 99DOTS would be used at treatment units in the absence of a research study.

\section{Randomisation}

The 18 health facilities will be randomly assigned to one of the six allocation sequences (figure 1) using a simple, unrestricted two-stage process. This process will occur by simple drawing during a public randomisation ceremony held in Kampala, Uganda. A representative from each TB treatment unit, district health officers and Uganda NTLP staff will be invited to attend the ceremony. First, health facilities (ie, clusters) will be randomly assigned into six blocks of three by having health facility representatives each draw 1 of 18 balls (three each labelled A-F) from an opaque bag. Blocks will then be randomly assigned to an intervention initiation time, which will occur at equally spaced 1-month intervals during the trial, by drawing of 6 balls labelled 1-6 from an opaque bag. 


\section{Blinding}

Blinding of the assigned intervention is not feasible given intervention implementation at the health facility level. Where possible, the investigators and study staff, with the exception of the statistician and data manager, will be blinded to aggregate TB outcomes by study period.

\section{Data collection and management}

Patient-level data collection

Consistent with the pragmatic design, $\mathrm{TB}$ treatment outcomes will be assessed by extracting data on all eligible patients from routine Uganda NTLP TB treatment registers used at all treatment units. Project staff will train two health workers at each site (one primary, one backup) identified by the health facility director to take photos of the register every 2-4 weeks for the duration of the project using a camera-enabled smartphone, and to upload the photos to a central secure, password-protected server, only accessible to staff. Health workers will be trained to delete photos from the phone after upload confirmation. Completeness of TB treatment registers will be assessed, and at quarterly site visits, study staff will provide retraining as needed and resolve data cleaning queries. Study staff will extract data from photos of TB registers and enter data into a secure database use Research Electronic Data Capture software (REDCap).${ }^{23}$ Data queries for missing or non-sensical data will be reviewed with treatment unit staff at quarterly site visits.

\section{D0TS process metrics data collection}

During the intervention period at each site, process metric data to assess the implementation of each component of the intervention strategy will be extracted from the 99DOTS server. The server logs all calls made by patients to confirm dosing and doses entered manually by providers, all SMS messages sent to patients, and all IVR calls sent to patients and their responses.

\section{Qualitative data collection}

Surveys will be conducted with 10 randomly selected eligible patients enrolled on 99DOTS (5 women and 5 men) and 1-2 eligible providers per site (180 total patients, 18-36 total providers) beginning in month 10 of the trial to assess the acceptability of the 99DOTS-based intervention strategy. Research staff will contact selected patients by phone to review the verbal consent script, answer questions the patient may have and administer the survey to consenting patients. Provider surveys and interviews will be conducted during quarterly site visits beginning in month 13 of the trial to assess factors influencing the adoption and implementation of 99DOTS.

\section{Health system cost data collection}

Costing and cost-effectiveness analyses will focus on the health system perspective. Treatment unit staff at each site will be interviewed to gain a more complete understanding of the activities and staff involved in the operations of 99DOTS. Time-and-motion studies of health workers (anticipated to be three to six health workers per site, depending on the number of staff involved in delivering 99DOTS) will be carried out at six clinics, sampled to ensure good representation of clinic volume and geography. These time-and-motion studies will be performed on a quarterly basis in conjunction with site visits and will consist of direct observation of all activities conducted by treatment unit staff over the corresponding 1-2 day evaluation period, for a total 6-12 days of observation every 3 months over the course of the 14-month study period. During these observation periods, the time and resources required to perform all activities related to TB treatment (eg, medication preparation, contacting patients, observing medication doses and so on) will be recorded. Additional costing data will be collected to assess the cost of implementing and maintaining 99DOTS technical assistance (budget records from Everwell Health Solutions, the creator of 99DOTS) and the cost at point of care (surveys of project staff to track resource use during trainings). Overhead costs will be estimated using an ingredients approach, incorporating the cost of supplies, building/space, vehicles and human resources with incorporation of recurrent costs such as security, vehicle maintenance costs and all supplies (medical and administrative).

\section{Outcomes}

Trial outcomes were selected in accordance with the RE-AIM evaluation framework (table 2). The primary effectiveness outcome is the proportion of patients who complete TB treatment, defined as having an outcome of 'cured' or 'treatment completed' recorded in the unit TB treatment register. Secondary effectiveness outcomes include the proportion who persist on treatment through the intensive phase (ie, 56 days of treatment), the proportion lost to follow-up and estimated number of incremental disability-adjusted life years averted. Reach is defined as the proportion of eligible patients enrolled on 99DOTS. Adoption metrics include the proportion of scheduled doses confirmed by patient phone calls and the proportion of weekly IVR check-in calls to which patients send a response. Implementation outcomes include delivery of the intervention (proportions of daily SMS dosing reminders and weekly IVR check-in calls sent by the 99DOTS platform and received on patient handsets), acceptability (as assessed by patient and health worker surveys and interviews) and incremental costs of the 99DOTS-based intervention strategy.

\section{Power and sample size}

The trial aims to demonstrate the superiority of the 99DOTS-based strategy. The sample size was calculated for the primary effectiveness outcome, proportion of patients treated successfully, using formulae appropriate for stepped-wedge trials. A type I error of $5 \%$ and power of at least $90 \%$ is assumed. Based on the 2017 data, the harmonic mean number of patients initiating treatment for drug-susceptible pulmonary TB per month across participating treatment units was 15 . Thus, we anticipate 
Table 2 Outcome definitions and data source by RE-AIM dimension

\begin{tabular}{|c|c|c|}
\hline Outcome type & Outcome & Data source \\
\hline \multicolumn{3}{|l|}{ Reach } \\
\hline \multicolumn{2}{|l|}{ Proportion enrolled on 99DOTS } & $\begin{array}{l}\text { 99DOTS server, treatment } \\
\text { register }\end{array}$ \\
\hline \multicolumn{3}{|l|}{ Effectiveness } \\
\hline Primary & Proportion treated successfully & Treatment register \\
\hline Secondary & $\begin{array}{l}\text { Incremental cost per patient treated } \\
\text { successfully }\end{array}$ & $\begin{array}{l}\text { Time and motion surveys; } \\
\text { budgetary analysis }\end{array}$ \\
\hline \multicolumn{3}{|l|}{ Adoption } \\
\hline \multicolumn{2}{|c|}{ Proportion of scheduled doses confirmed by phone call } & 99DOTS server \\
\hline \multicolumn{2}{|c|}{ Proportion of daily SMS received on patient handset } & 99DOTS server \\
\hline \multicolumn{2}{|c|}{ Proportion of weekly IVR calls sent by 99DOTS platform } & 99DOTS server \\
\hline \multicolumn{2}{|c|}{ Proportion of weekly IVR calls received on patient handset } & 99DOTS server \\
\hline
\end{tabular}

DOTS, directly observed therapy short-course; IVR, interactive voice response.

that approximately 1890 patients will initiate treatment over the 8-month enrolment period (945 in the preimplementation and 945 in the postimplementation phases across treatment units). The trial will have $89 \%$ power to demonstrate that our strategy increases the proportion of patients treated successfully by $10 \%$ or more (assumptions: alpha $=0.05$; intraclass correlation coefficient, ICC $=0.001$ calculated using 2017 NTLP data for the 18 treatment units; preimplementation treatment success $=51 \%$ based on the 2017 NTLP data for the 18 treatment units; calculations performed using steppedwedge command in Stata V.14).

\section{Analysis}

The primary effectiveness analysis will be conducted at the health facility level using multivariable mixed effect logit models with random effects for site and fixed effects for trial period, time and confounders (using Stata's melogit and meqrlogit commands). Analysis will be done for intention-to-treat (all eligible patients) and per protocol (excluding patients who are not enrolled on 99DOTS during the intervention period at each site) populations. Models will adjust for the longitudinal design (indicator variable for each trial month) and clustering by site (random effect for health facility). Patients initiating treatment during the month in which sites switch from routine treatment supervision to the intervention strategy will be excluded (buffer period). Potential confounders, selected a priori, including sex, HIV status, disease class (bacteriologically confirmed vs clinically diagnosed) and TB type (new vs retreatment), will be included in the model as fixed effects. Secondary effectiveness outcomes (table 2) will be analysed in the same manner. Subgroup analyses will stratify by gender, HIV status and health facility. To further assess the robustness of our findings, we will conduct sensitivity analyses that include patients who initiated treatment during the buffer period, impute outcomes for patients lost to follow-up and compare treatment outcomes using permutation tests. ${ }^{24}$

Quantitative reach, adoption and implementation outcomes will be summarised descriptively. Comparative analyses will identify factors independently associated with reach, adoption and implementation of intervention components. Health economic data will be used to calculate the incremental cost-effectiveness of the intervention strategy from a health system perspective, measured as the incremental cost per successfully completed treatment, comparing 99DOTS relative to routine care.

\section{Ethics and dissemination}

Patients can choose to use or not use 99DOTS at any time during the intervention period. 99DOTS enables closer patient monitoring than routine TB treatment supervision, but loss of patient privacy is a potential concern. Data on this are being collected through patient surveys. Because of the low-risk nature of the research, the principal investigators will be responsible for monitoring the data, assuring protocol compliance and conducting safety reviews on a quarterly basis. External monitoring is provided by a Stop TB Partnership project officer and monitoring and evaluation consultant. The principal investigator will submit regular progress reports, including recommendations on whether the project 
should continue unchanged, require modification or close to enrolment.

Ethics approval was granted by institutional review boards (IRBs)at Makerere University School of Public Health and the University of California San Francisco. To ensure that the study captures all eligible adults initiating treatment at study sites, a waiver of informed consent was granted to access routine TB treatment data recorded in standard NTLP registers, such that research staff will not be required to be onsite to enrol and consent patients. The protocol was registered with the Pan-African Clinical Trials Registry (PACTR201808609844917) on 31 August 2018, updated 7 November 2019 and complies with reporting guidelines outlined in the stepped-wedge trial extension of the Consolidated Standards of Reporting Trials. ${ }^{25}$ Any major changes in protocol will be approved by the Stop TB Partnership project officer and both ethics committees and registered with the PACTR. The full protocol and statistical code will be made available on request.

Findings will be disseminated through peer-reviewed publications, presentations at scientific conferences and presentations to key stakeholders. Drs Cattamanchi, Katamba and Kiwanuka will have access to the final dataset; the participant-level dataset will be made available to other investigators who have IRB approval to analyse the data.

\section{Patient and public involvement}

Patients and members of the public were involved throughout the research process. The NTLP was involved in identifying health facilities to participate, selecting study design and conducting research through partnership with District Health Officers and participating health facilities. Patients, health workers and community members were involved in intervention design through a human-centred design process, which included several rounds of focus groups and interviews with stakeholders. Health workers at the 18 health facilities implement 99DOTS without research staff present and assist in data collection by completing routine TB treatment registers and sending photos of the register to the research team through secure means.

\section{DISCUSSION}

99DOTS is a low-cost DAT that has the potential to improve TB treatment outcomes and the patient experience of TB treatment in Uganda and other high-burden settings. Here, we describe the first randomised trial designed to evaluate the effectiveness of a 99DOTS-based TB treatment supervision strategy as part of routine care in a high-burden setting. Other novel aspects of the trial include our use of human-centred design to adapt 99DOTS to the local context with the goal of increasing patient engagement with the technology, the use of theory and implementation science frameworks to guide intervention design and evaluation, and our focus on simultaneously assessing implementation and costs to guide scale-up decisions.

Many aspects of this trial are intentionally pragmatic to ensure the outcomes reflect what can be expected under non-research conditions. ${ }^{20}$ The study population will include all patients with drug susceptible pulmonary TB, with the exception of children. A waiver of consent was obtained for patient-level data collection such that research staff will not be required to be onsite to enrol and consent patients. Primary and key secondary outcomes will be assessed using routine data available through NTLP registers and the 99DOTS platform, with focused additional data collection for implementation and cost outcomes. The intervention will be implemented by routine $\mathrm{TB}$ treatment unit staff, who also will make all decisions to offer 99DOTS-based treatment supervision to patients. At the same time, the stepped-wedge randomised trial design provides a rigorous assessment of intervention effect. Limitations of such a pragmatic trial design include less control over intervention delivery, potential limited uptake of the intervention given the wide inclusion criteria (all adults initiating treatment for drug-susceptible pulmonary $\mathrm{TB}$ ) and inability to rigorously verify medication adherence during the control and intervention periods. In order to enrol enough patients to assess the effectiveness of the 99DOTS-based intervention, we selected facilities that treat larger numbers of patients with TB. Uptake and effectiveness of 99DOTS may be different at lower volume health centres.

In summary, this pragmatic, hybrid type 2 effectivenessimplementation trial is well poised to assess both the effectiveness of an adapted 99DOTS-based intervention and potential barriers and facilitators to its scale-up if successful. The design and implementation of this trial intend to generate results to inform decisions on whether and how to implement 99DOTS in Uganda and other high-burden countries.

\section{Author affiliations}

${ }^{1}$ Center for Tuberculosis and Division of Pulmonary and Critical Care Medicine, San Francisco General Hospital, University of California San Francisco, San Francisco, California, USA

${ }^{2}$ Uganda Tuberculosis Implementation Research Consortium, Kampala, Uganda ${ }^{3}$ School of Public Heatlh, Makerere University College of Health Sciences, Kampala, Uganda

${ }^{4}$ Department of Epidemiology, Johns Hopkins University Bloomberg School of Public Health, Baltimore, Maryland, USA

${ }^{5}$ The Better Lab, Department of Surgery, Zuckerberg San Francisco General Hospital, University of California San Francisco, San Francisco, California, USA ${ }^{6}$ National Tuberculosis and Leprosy Program, Republic of Uganda Ministry of Health, Kampala, Uganda

${ }^{7}$ School of Medicine, Makerere University College of Health Sciences, Kampala, Uganda

Acknowledgements We would like to thank the patients and health facility staff who make this research possible, as well as the Uganda NTLP, for their collaboration on this protocol.

Contributors AC, AKa and NK conceived and designed the study. RC, AKi, ML, CN, LKT, ASN, JG, PT, DB, D0, CAB, AT, DP, AS, DD, TS, AC and AKa participated in the implementation of the study. RC, AKi, AC and AKa drafted the manuscript. All authors reviewed and approved the manuscript. 
Funding This Project is supported by the Stop TB Partnership's TB REACH initiative, grant number STBP/TBREACH/GSA/W6-37, which is funded by the Government of Canada, the Bill \& Melinda Gates Foundation, and the United States Agency for International Development. The study sponsor is not involved in data collection, analysis or interpretation.

Competing interests None declared.

Patient and public involvement Patients and/or the public were involved in the design, or conduct, or reporting or dissemination plans of this research. Refer to the Methods section for further details.

Patient consent for publication Not required.

Provenance and peer review Not commissioned; externally peer reviewed.

Open access This is an open access article distributed in accordance with the Creative Commons Attribution Non Commercial (CC BY-NC 4.0) license, which permits others to distribute, remix, adapt, build upon this work non-commercially, and license their derivative works on different terms, provided the original work is properly cited, appropriate credit is given, any changes made indicated, and the use is non-commercial. See: http://creativecommons.org/licenses/by-nc/4.0/.

ORCID iDs

Christopher Allen Berger http://orcid.org/0000-0002-0034-7544

Achilles Katamba http://orcid.org/0000-0002-2347-4183

\section{REFERENCES}

1 World Health Organization. Global tuberculosis report; 2019.

2 Izudi J, Tamwesigire IK, Bajunirwe F. Explaining the successes and failures of tuberculosis treatment programs; a tale of two regions in rural eastern Uganda. BMC Health Serv Res 2019;19:979.

3 Fiseha D, Demissie M. Assessment of directly observed therapy (dot) following tuberculosis regimen change in Addis Ababa, Ethiopia: a qualitative study. BMC Infect Dis 2015;15:405.

4 Intelligence G. The Mobile Economy - Africa London: GSMA; 2016. Available: https://www.gsma.com/mobileeconomy/africa/

5 World Health Organization. Treatment of tuberculosis: guidelines for treatment of drug-susceptible tuberculosis and patient care (2017 update). Geneva, 2017.

6 Liu X, Lewis JJ, Zhang H, et al. Effectiveness of electronic reminders to improve medication adherence in tuberculosis patients: a clusterrandomised trial. PLoS Med 2015;12:e1001876-e.

7 Subbaraman R, de Mondesert L, Musiimenta A, et al. Digital adherence technologies for the management of tuberculosis therapy: mapping the landscape and research priorities. BMJ Glob Health 2018;3:e001018.

8 Liu X, Lewis JJ, Zhang H, et al. Effectiveness of electronic reminders to improve medication adherence in tuberculosis patients: a clusterrandomised trial. PLoS Med 2015;12:e1001876.
9 Liu X, Blaschke T, Thomas B, et al. Usability of a medication event reminder monitor system (MERM) by providers and patients to improve adherence in the management of tuberculosis. Int $J$ Environ Res Public Health 2017;14. doi:10.3390/ijerph14101115. [Epub ahead of print: 2509 2017].

10 Thakkar D, Piparva KG, Lakkad SG. A pilot project: 99DOTS information communication technology-based approach for tuberculosis treatment in Rajkot district. Lung India 2019;36:108-11.

11 Cross A, Gupta N, Liu B. 99DOTS: a low-cost approach to monitoring and improving medication adherence. Tenth international conference on information and communication technologies and development. ICTD, 2019.

12 Green LW, Kreuter MW. Health promotion planning: an educational and ecological approach. McGraw-Hill, 1993.

13 Atkins L, Francis J, Islam R, et al. A guide to using the theoretical domains framework of behaviour change to investigate implementation problems. Implement Sci 2017;12:77.

14 French SD, Green SE, O'Connor DA, et al. Developing theoryinformed behaviour change interventions to implement evidence into practice: a systematic approach using the theoretical domains framework. Implement Sci 2012;7:38.

15 Sarfaraz J. Unified theory of acceptance and use of technology (Utaut) model-mobile banking. JIBC 2017;22:1-20.

16 Venkatesh V, Thong JY, Xu X. Consumer acceptance and use of information technology: extending the unified theory of acceptance and use of technology. MIS Quarterly 2012;36:157-78.

17 Glasgow RE, Vogt TM, Boles SM. Evaluating the public health impact of health promotion interventions: the RE-AIM framework. Am J Public Health 1999;89:1322-7.

18 Gaglio B, Shoup JA, Glasgow RE. The RE-AIM framework: a systematic review of use over time. Am J Public Health 2013;103:e38-46.

19 Curran GM, Bauer M, Mittman B, et al. Effectiveness-implementation hybrid designs: combining elements of clinical effectiveness and implementation research to enhance public health impact. Med Care 2012;50:217-26.

20 Loudon K, Treweek S, Sullivan F, et al. The PRECIS-2 tool: designing trials that are fit for purpose. BMJ 2015;350:h2147.

21 Ggita JM, Ojok C, Meyer AJ, et al. Patterns of usage and preferences of users for tuberculosis-related text messages and voice calls in Uganda. Int J Tuberc Lung Dis 2018;22:530-6.

22 Babirye D, Shete PB, Farr K, et al. Feasibility of a short message service (SMS) intervention to deliver tuberculosis testing results in peri-urban and rural Uganda. J Clin Tuberc Other Mycobact Dis 2019;16:100110.

23 Harris PA, Taylor R, Minor BL, et al. The REDCap Consortium: building an international community of software platform partners. $J$ Biomed Inform 2019;95:103208.

24 Thompson J, Davey C, Hayes R, et al. swpermute: permutation tests for Stepped-Wedge cluster-randomised trials. Stata J 2019;19:803-19.

25 Hemming K, Taljaard M, McKenzie JE, et al. Reporting of stepped wedge cluster randomised trials: extension of the CONSORT 2010 statement with explanation and elaboration. BMJ 2018;363:k1614. 